\title{
Leptosphaeria senegalensis : \\ Facteurs optimums de sa sexualisation
}

\section{par S. ANDRIEU (*), F. TRAORE (**), L. DUJARDIN (*) et L. LACOSTE (***)}

(Collaboration technique : R. POPEYe $\left({ }^{*}\right)$

* Unité I.N.S.E.R.M. (U. 42), Domaine du C.E.R.T.I.A., 369, rue Jules-Guesde, Flers-Bourg, 59650 Villeneuve-d'Ascq, France.

** Laboratoire de Parasitologie, Faculté de Médecine, 15, rue de l'Ecole-de-Médecine, 75006 Paris.

**** Laboratoire de Cryptogamie, Université des Sciences et Techniques, 59655 Villeneuve-d'Ascq, Cedex, France.

\section{Résựé.}

Parmi divers milieux "naturels » incubés dans les conditions empiriques habituelles, est sélectionné celui qui permet la meilleure fructification sexuée de L.s. Les conditions optimales de nature de la lumière, de temps d'éclairement, de température d'incubation sont déterminées sur ce milieu: à la lumière blanche, appliquée $12 \mathrm{~h}$ dans le nycthémère et à la température de 22 à $24^{\circ} \mathrm{C}$, les ascospores mûrissent au bout de 25 jours.

Dans ces conditions d'incubation est recherché le milieu synthétique capable de permettre la reproduction sexuée. Le meilleur équilibre $\mathrm{C} / \mathrm{N}$ permettant sexualisation et maturation des ascospores, est recherché ensuite mais ne peut être précisément défini. Par contre, un "milieu de routine» composé sur ces données et incubé dans les conditions déterminées, permet sexualisation et maturation en 18 jours.

\section{Summiary.}

\section{Leptosphaeria senegalensis : Sexualisation optimum factors.}

Among various "natural " media incubated in the usual experimental conditions, the one that enables the sexual fructification of Leptosphaeria senegalensis is chosen.

The optimal conditions of light nature, lightning time, incubation temperature, are determined for this medium : white light, during 12 hours in the nyctemere at $22^{\circ}-24^{\circ} \mathrm{C}$ enables the ascospore maturation after 25 days.

Accepté le 30 octobre 1979. 
In these incubation conditions, a synthetic medium allowing the best sexual reproduction is made up. But the best $\mathrm{C} / \mathrm{N}$ equilibrium to obtain sexualisation and ascospore maturation cannot be exactly defined on the other hand, a routine medium constitued on the data and incubated in these determined conditions gives sexualisation and maturation after 18 days.

Parmi les agents des mycétomes africains, Leptosphaeria senegalensis est une espèce bien individualisée : elle donne en culture sa forme sexuée, décrite par ses inventeurs (1) comme étant un «Ascomycète, produisant sur certains milieux des asques enfermés dans des périthèces et contenant 8 ascospores septées ». La description des périthèces conduit les auteurs à ranger cette espèce dans la sous-classe des Pyrénomycètes, Pseudosphaeriales, Pleosporaceae, genre Leptosphaeria.

Mais son attribution au genre Leptosphaeria l'a été avec grande prudence par les inventeurs (1) et est mise en doute par d'autres (5). Pour affiner son identification, étant donné que son biotope naturel, en dehors des mycétomes, est ignoré, il nous apparaît opportun de mieux connaître l'ontogénie de sa forme sexuée.

Or, d'après les expérimentateurs qui ont cultivé cette espèce (1), cette forme sexuée se différencie souvent très tardivement, voire même jamais sur les milieux couramment utilisés. Enfin, suivant les techniques adoptées la maturation des ascospores peut être très tardive ou impossible à obtenir.

C'est pourquoi nous nous sommes attachés à définir les conditions optimales d'obtention du développement complet du Leptosphaeria senegalensis en vue d'obtenir une production abondante et régulière d'ascocarpes pour en étudier ultérieurement l'ontogénie.

\section{A. - Choix d'un milieu naturel}

La méconnaissance du biotope naturel du L. senegalensis trouvé sur les épines des arbustes colonisant les bords des chemins sans qu'on puisse dire qu'il vit réellement sur ces plantes, nous empêche d'utiliser la méthode préconisée par l'un de nous (5) pour le choix du milieu de référence : la plante-hôte. Par suite les milieux utilisés jusqu'ici pour isoler et cultiver cette espèce sont des milieux classiques en mycologie médicale: gélose de Sabouraud glucosée ou maltosée, moût de bière gélosé, gélose peptonée non sucrée, Czapek, pommes de terre - carottes glucosées (P.C.) et surtout le milieu pommes de terre - carottes maltea (P.C. maltea) à raison de $40 \mathrm{~g}, 40 \mathrm{~g}$ et $5 \mathrm{~g}$ respectivement par litre de milieu gélosé.

Dans ces conditions nutritives, aucun auteur n’a à ce jour précisé la température et l'éclairement convenables et la plupart se contente de laisser les cultures se développer à la température ambiante "sur la paillasse ou le bureau ". Le délai d'obtention de périthèces mûrs est alors très variable : 2 à 4 mois, ou plus. D'autres mycologues n'en obtiennent jamais dans de telles conditions.

Pour déterminer les conditions physiologiques optimales de la sexualisation de cette espèce, nous devions d'abord choisir, parmi des milieux non chimiquement 
définis, ou "naturels", celui qui donnerait une fructification arrivant à maturité, dans les conditions empiriques traditionnelles; soumettre ensuite les repiquages à un éventail de conditions d'incubation nous permettant de préciser le rôle de la température et de l'éclairement et d'en fixer les optimums.

\section{1. - Matériel et méthodes.}

Nous avons choisi deux souches : 630 I.P.P. et F..., la première conservée depuis plusieurs années au laboratoire, la deuxième récemment isolée d'un mycétome de la hanche, toutes deux cultivées sur P.C. maltea.

Les milieux proposés au champignon ont été comparativement au Czapek, Sabouraud et P.C. maltea successivement le moût de bière gélosé, dilué au $1 / 2,1 / 4,1 / 8^{\mathrm{e}}$, $1 / 16^{e}$, le P.C. à partir de variétés différentes de légumes, les morceaux de pommes de terre et carottes en tubes, diverses tiges et feuilles de graminées ( foin » commun) mais plus spécialement celles de Phragmites communis, ainsi que les décoctions gélosées de ces plantes, à raison de 100,50 et $25 \mathrm{~g} /$ litre de «foin » sec.

L'ensemencement a été fait à partir d'ascospores ou de fragments de mycélium.

L'incubation a eu lieu sur la paillasse, la température ambiante variant de 18 à $25^{\circ} \mathrm{C}$ suivant les jours et les saisons.

\section{2. - Résultats.}

Les mêmes délais, très longs ( 2 à 3 mois) ou plus souvent l'échec, sont venus confirmer la difficulté d'obtenir des périthèces mûrs sur ces milieux dans ces conditions.

Seuls les milieux à base de Phragmites ont été satisfaisants. Nous avons vu apparaître en 3 semaines des périthèces en surface des tiges et des feuilles, mais aussi en surface et en profondeur de la décoction gélosée, à la concentration de $50 \mathrm{~g}$ de plante sèche par litre. La măturation des périthèces situés en surface est obtenue en 45 jours. Nombreux sont les périthèces qui, en profondeur de la gélose, restent immatures. Tous nos repiquages se conduisant de façon identique, nous décidons d'adopter ce milieu à base de Phragmites, comme milieu naturel de référence pour nos expérimentations ultérieures.

\section{B. - Recherche des conditions optimales d'éclairement et de température}

Au cours de l'étude biologique des Leptosphaeria vrais, l'un de nous (5) a pu montrer qu'existe une écologie propre à chaque espèce, et notamment des exigences de températures et d'éclairements particulières. Ces facteurs méritaient d'être étudiés pour notre espèce. 


\section{1. - Matériel et méthodes.}

Nos étuves obscures nous ont permis d'évaluer les limites de température entre lesquelles une croissance mycélienne est possible.

Nous avons ensuite disposé d'enceintes conditionnées à hygrométrie fixe, dont les systèmes de régulation permettent de maintenir l'ambiance interne à des températures comprises entre $10^{\circ} \mathrm{C}$ et $30^{\circ} \mathrm{C}$, à $\pm 0,5^{\circ} \mathrm{C}$ près, et dont le système d'éclairement est constitué, pour la lumière blanche, par des tubes fluorescents «blanc brillant de luxe » donnant sur la surface de culture, une énergie de $750 \mu \mathrm{W} / \mathrm{cm}^{2}$. Un compteur horaire disposé en série sur l'alimentation des tubes permettait de faire varier à volonté le rythme des éclairements à alterner avec l'obscurité.

Avec des tubes fluorescents colorés, donnant des lumières largement monochromatiques, on obtient des éclairements en lumière proche ultra-violette (lumière noire), bleue, verte ou rouge, avec des énergies équivalentes aux précédentes.

\section{2. - Résultats.}

a) Température :

- une croissance mycélienne n'apparaît pratiquement plus en-deçà de $15^{\circ} \mathrm{C}$ et au-delà de $40^{\circ} \mathrm{C}$;

- la sexualisation s'effectue au-dessus de $20^{\circ} \mathrm{C}$ et au-dessous de $35^{\circ} \mathrm{C}$, mais la mäturation des ascospores ne s'observe pas aux températures extrêmes; le nombre maximum de périthèces capables d'arriver à máturité s'obtient entre 22 et $24^{\circ} \mathrm{C}$.

b) Eclairement :

- la croissance mycélienne est plus abondante, aux mêmes températures, à l'obscurité totale qu'à la lumière blanche. Les lumières noire, verte ou rouge ont sensiblement la même influence que l'obscurité. La lumière bleue permet une croissance mycélienne semblable à celle obtenue en lumière blanche ;

- la sexualisation est absente à l'obscurité totale, et cela même si l'on maintient les cultures en incubation pendant plusieurs mois à $22{ }^{\circ} \mathrm{C}$.

L'application de la lumière blanche, même pendant un temps très court (1 heure), provoque un début de sexualisation, mais celle-ci est optimale en nombre et en màturation lorsque cette lumière est appliquée 12 heures dans le nycthémère pendant toute la culture.

La lumière bleue permet le début de la sexualisation mais non la maturation (seules, de rares ébauches peuvent apparaître); en lumière verte, rouge, ou proche U.V., la réponse est identique à celle obtenue à l'obscurité totale : aucun périthèce n'apparaît, même au simple stade d'ébauche.

En conclusion, la sexualisation et la maturation sont optimales entre 22 et $24^{\circ} \mathrm{C}$, et à un éclairement de lumière blanche de $12 \mathrm{~h}$ dans le nycthémère; 
- enfin, il faut noter que les cultures maintenues strictement à l'obscurité ne fructifient pas, même au bout de 6 à 7 mois (limite de notre expérience). Les anastomoses existent mais n'évoluent pas. Par contre, et quel que soit l'âge de la culture, si après avoir été maintenue à cette stricte obscurité, elle est exposée à la lumière blanche pendant la moitié du nycthémère, elle fructifie très rapidement, et les premières ascospores mûres apparaissent 5 jours après. Dans ces conditions, les périthèces profondément situés dans la gélose semblent mûrir aussi rapidement que les périthèces de surface.

\section{C. - Détermination d'un milieu synthétique permettant croissance et sexualisation}

\section{1. - Matériel et méthodes.}

Nous inspirant de travaux antérieurs $(2,4)$, nous avons utilisé un milieu minéral (m.m.) convenant à la sexualisation des Dermatophytes (Gymnoascaceae), et composé comme suit pour un litre :

$\mathrm{KH}_{2} \mathrm{PO}_{4}: 1,5 \mathrm{~g} ; \mathrm{MgSO}_{4}, 7 \mathrm{H}_{2} \mathrm{O}: 0,5 \mathrm{~g} ; \mathrm{CaCl}_{2}, 2 \mathrm{H}_{2} \mathrm{O}: 0,15 \mathrm{~g}$; $\mathrm{ZnSO}_{4}, 7 \mathrm{H}_{2} \mathrm{O}: 0,020 \mathrm{~g}$; $\mathrm{CuSO}_{4}, 5 \mathrm{H}_{2} \mathrm{O}: 0,010 \mathrm{~g} ; \mathrm{MnSO}_{4}, \mathrm{H}_{2} \mathrm{O}: 0,015 \mathrm{~g}$.

auquel nous avons apporté un sucre à concentration fixe: le giucose, a raison de $10 \mathrm{~g} /$ litre, et les vitamines suivantes, seules ou en mélange de 2, 3, 4 ou 5 éléments pour 1 litre :

Biotine : $5 \mu \mathrm{g}$; Thiamine $100 \mu \mathrm{g}$; Acide nicotinique : $100 \mu \mathrm{g}$; Pantothénate de $\mathrm{Ca}: 200 \mu \mathrm{g}$; Pyridoxine, $\mathrm{HCl}: 100 \mu \mathrm{g}$.

Nous avons essayé, comme source d'azote:

— soit un acide aminé : sérine, ou acide glutamique, ou acide aspartique, à raison de $100 \mathrm{mg}$ d'azote par litre ;

- soit un composé minéral azoté : $\left(\mathrm{NH}_{4}\right)_{2} \mathrm{SO}_{4}$ ou $\mathrm{KNO}_{3}$ à la même concentration.

Après avoir déterminé la meilleure source $\mathrm{d}$ ' $\mathrm{N}$, nous avons proposé au champignon les sucres suivants, substitués au glucose dans des proportions croissantes de $0,5 \mathrm{~g}$ à $2 \mathrm{~g} /$ litre, le milieu au glucose servant de témoin, aux mêmes dilutions : D- fructose, Maltose, Saccharose, Lactose et $\mathrm{D}^{+}$xylose.

\section{2. - Résultats.}

a) le milieu minéral choisi convient parfaitement à la croissance, puis à la sexualisation de $L$. senegalensis, si on lui apporte sucre, azote et vitamines convenables;

b) l'azote minéral ne convient ni à la croissance [très faible avec $\mathrm{KNO}_{3}$, nulle avec $\left.\left(\mathrm{NH}_{4}\right)_{2} \mathrm{SO}_{4}\right]$, ni à la sexualisation ; 
c) les trois acides aminés testés ensemble à part égale n'ont pas donné une croissance supérieure à celle obtenue sur la sérine utilisée seule. Le développement reste médiocre sur l'acide glutamique et sur l'acide aspartique et toujours inférieur à celui obtenu avec la sérine ;

d) le glucose est le sucre le plus favorable à la croissance puis à la sexualisation, et la concentration optimale est de $0,5 \mathrm{~g} /$ litre dans les conditions et le milieu minéral donnés ;

e) certaines vitamines sont indispensables à une bonne croissance et à la sexualisation. Le complexe vitaminique le meilleur est le suivant: Biotine $5 \mu \mathrm{g} / 1$ et Thiamine $100 \mu \mathrm{g} / 1$.

La formule exacte du milieu ainsi déterminé est donc:

$\mathrm{KH}_{2} \mathrm{PO}_{4}: 1,5 \mathrm{~g} / 1 ; \mathrm{MgSO}_{4}, 7 \mathrm{H}_{2} \mathrm{O}: 0,5 \mathrm{~g} / 1 ; \mathrm{CaCl}_{2}, 2 \mathrm{H}_{2} \mathrm{O}: 0,15 \mathrm{~g} / 1$;

$\mathrm{ZnSO}_{4}, 5 \mathrm{H}_{2} \mathrm{O}: 0,020 \mathrm{~g} / 1 ; \mathrm{CuSO}_{4}, 5 \mathrm{H}_{2} \mathrm{O}: 0,010 \mathrm{~g} / 1 ; \mathrm{MnSO}_{4}, \mathrm{H}_{2} \mathrm{O}: 0,015 \mathrm{~g} / 1$;

glucose : $0,5 \mathrm{~g} / 1$; sérine $(50 \mathrm{mg} \mathrm{N} / \mathrm{l}): 0,375 \mathrm{~g} / 1$; biotine : $5 \mu \mathrm{g} / 1$; thiamine : $100 \mu \mathrm{g} / 1$.

$\mathrm{Au}$ terme de cette expérimentation, dans les conditions physiques déterminées au paragraphe précédent, ce milieu permet la maturation des ascospores de $L$. senegalensis en 25 jours.

\section{D. - Recherche du meilleur équilibre $\mathrm{C} / \mathrm{N}$}

\section{permettant la sexualisation et la maturation des ascospores}

Les travaux de Westegaard et coll. (7), de Hall (3) et de Lacoste et coll. (4) ont montré que les périthèces de Neurospora sp., de Sordaria fimicola et de Arachniotus albicans sont produits sur des milieux de culture ayant le même rapport $\mathrm{C} / \mathrm{N}$ et que les quantités absolues de $\mathrm{C}$ et de $\mathrm{N}$ pouvaient varier dans certaines limites pourvu que le rapport $\mathrm{C} / \mathrm{N}$ soit conservé.

Donc, dans un tableau à double entrée (fig. 1) où les milieux sont classés selon leur teneur en $\mathrm{C}$ et $\mathrm{N}$, les milieux favorables à la reproduction sexuée sont situés sur un segment d'une droite où s'inscrivent l'ensemble des milieux ayant le même rapport $\mathrm{C} / \mathrm{N}$.

Pour rechercher les milieux favorables à la reproduction sexuée, il faut donc réaliser un certain nombre de milieux du tableau à double entrée sans savoir si l'on se trouvera dans une partie intéressante du tableau (méthode 1).

$\mathrm{Si}$ nous émettons l'hypothèse supplémentaire que le rapport $\mathrm{C} / \mathrm{N}$ favorable à la reproduction sexuée est celui permettant une croissance équilibrée du champignon, un milieu sans excès ni de carbone, ni d'azote, c'est-à-dire un milieu qui, pour une quantité fixée arbitrairement d'azote (ou de carbone), contient la quantité minimale de carbone (ou d'azote) pour obtenir une croissance maximale du champignon, nous pouvons envisager une autre méthode de recherche (méthode 2).

Il suffit, en effet, de mesurer la croissance du champignon dans des milieux liquides situés dans une colonne ou une rangée du tableau I pour déterminer ce 
rapport $\mathrm{C} / \mathrm{N}$ équilibré pour la croissance; il suffit ensuite d'étudier l'ensemble des milieux ayant même rapport $\mathrm{C} / \mathrm{N}$ pour découvrir le segment sur lequel on observe la reproduction sexuée.

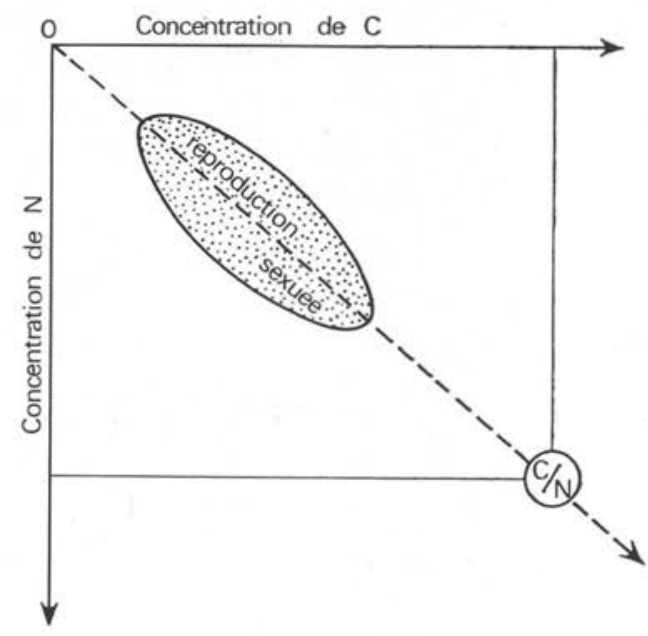

Fig. 1. Schématisation des méthodes de recherche de l'équilibre $\mathrm{C} / \mathrm{N}$ favorable à la reproduction sexuée.

Quelle que soit la quantité de carbone (ou d'azote) fixée arbitrairement au début de la recherche, la méthode aboutit obligatoirement à trouver un rapport $\mathrm{C} / \mathrm{N}$, alors qu'avec la méthode 1 , selon les limites des quantités absolues de $\mathrm{C}$ et de $\mathrm{N}$ choisies pour l'expérimentation, on observera ou non la reproduction sexuée.

Nous avons utilisé les deux méthodes pour préciser le rapport $\mathrm{C} / \mathrm{N}$ le plus favorable à la reproduction sexuée et pour tenter de vérifier notre hypothèse en utilisant le milieu de culture synthétique défini au paragraphe précédent, dans lequel on fait varier les concentrations de glucose et de sérine.

Les courbes de la figure 2 montrent que le rapport $\mathrm{C} / \mathrm{N}$, pour obtenir une croissance équilibrée du champignon telle que nous l'avons définie précédemment, correspond à $5 \mathrm{~g} / 1$ de glucose pour $50 \mathrm{mg} \mathrm{N} / 1$ (apportés par $0,375 \mathrm{~g} / 1$ de sérine).

La figure 3 indique les milieux favorables à la reproduction sexuée. On constate qu'ils ont des rapports $\mathrm{C} / \mathrm{N}$ différents de ceux trouvés comme optimums pour la croissance.

Il est remarquable que les périthèces de $L$. senegalensis apparaissent dans les milieux contenant un excès d'azote. L'hypothèse que nous avions formulée n'est donc pas vérifiée pour $L$. senegalensis et il faut constater que ce champignon ne fructifie pas sexuellement pour un rapport $\mathrm{C} / \mathrm{N}$ bien précis. Il serait pourtant intéressant de 
vérifier notre hypothèse avec des champignons tels que Neurospora sp., Sordaria fimicola ou Arachniotus albicans, déjà cités.

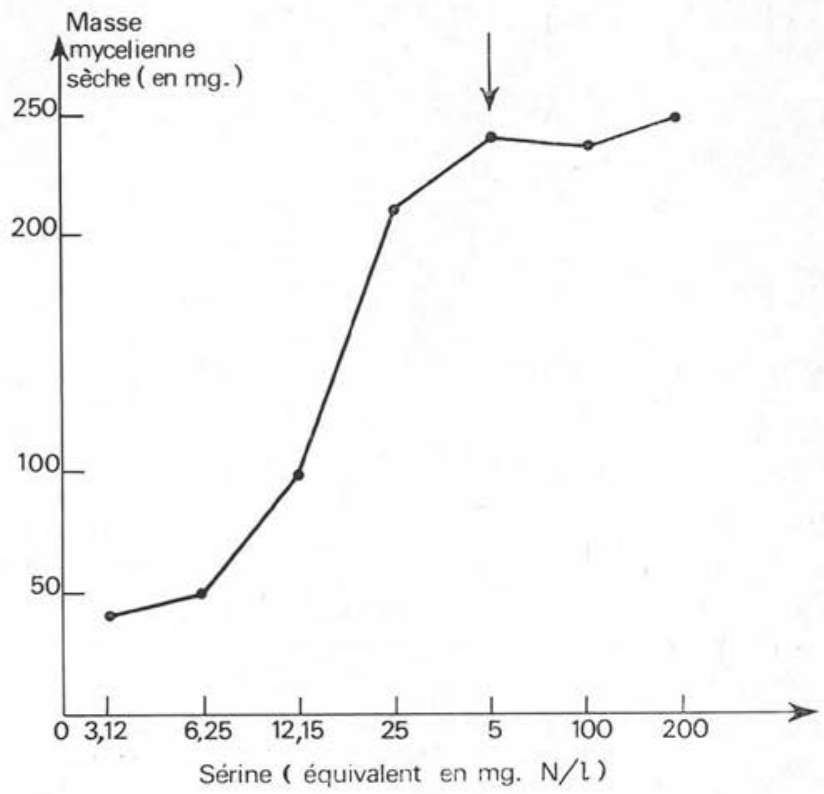

Fig. 2. Croissance mycélienne, au $21^{\circ}$ jour, de L.s. sur milieu synthétique contenant des doses croissantes de Sérine et une dose fixe de Glucose $(5 \mathrm{~g} / \mathrm{l})$.

\section{E. - Composition d'un milieu de routine, application pratique des recherches précédentes}

Le milieu ci-dessus défini peut difficilement être conseillé, pour un usage de routine, à des laboratoires dont l'installation "sur le terrain " peut être précaire. On peut sans doute composer, avec des produits plus banaux, un milieu respectant l'équilibre $\mathrm{C} / \mathrm{N}$ défini plus haut favorisant la sexualisation.

En utilisant des produits courants comme la peptone et le glucose, nous proposons la formule suivante pour 1 litre d'eau:

glucose : $2 \mathrm{~g}$; Néopeptone : $0,7 \mathrm{~g} ; \mathrm{KH}_{2} \mathrm{PO}_{4}: 1,5 \mathrm{~g} ; \mathrm{MgSO}_{4}, 7 \mathrm{H}_{2} \mathrm{O}: 0,5 \mathrm{~g}$; glucose : $20 \mathrm{~g}$.

On a la surprise de découvrir, après incubation en lumière blanche et obscurité $(12 \mathrm{~h}-12 \mathrm{~h})$ et à $22^{\circ} \mathrm{C}$, des périthèces mûrs en 18 jours, de façon systématique. Il resterait à analyser quels éléments, dans ce milieu complexe, accélère ainsi la maturation sexuelle. 


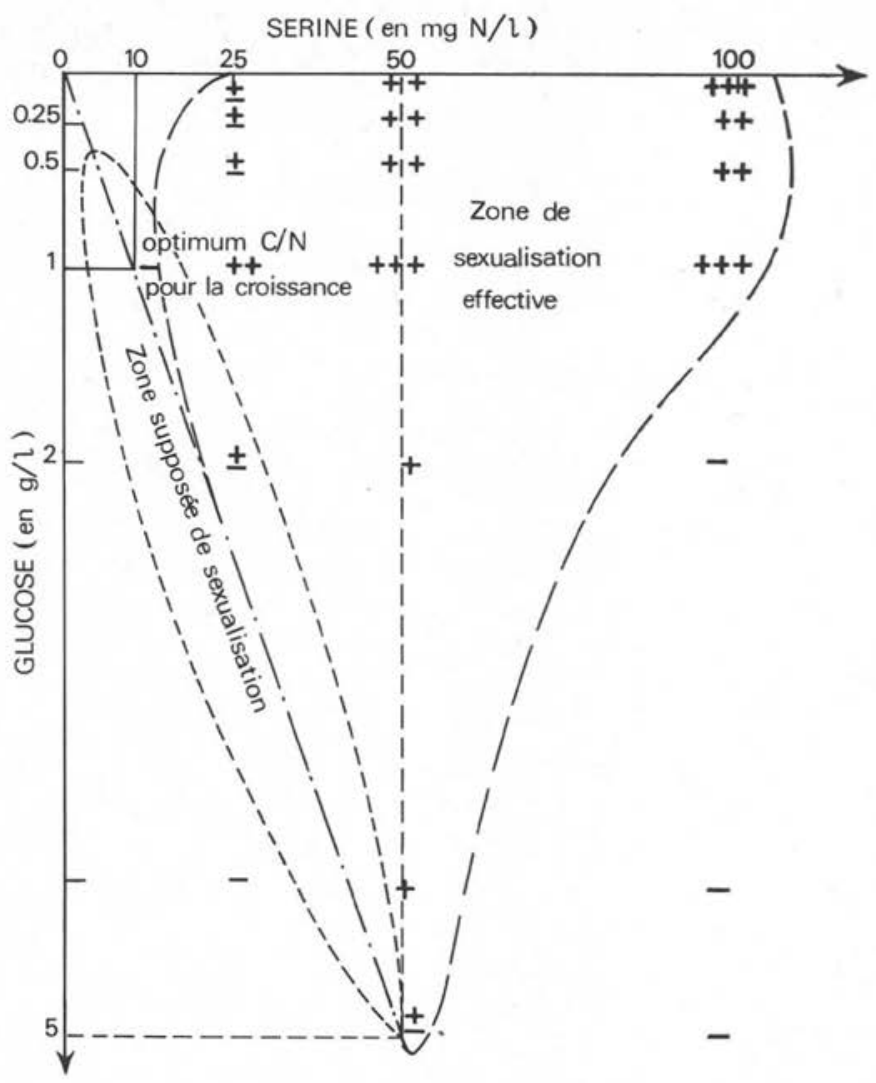

Fig. 3. Milieux favorables à la reproduction sexuée en fonction du rapport $\mathrm{C} / \mathrm{N}$.

Remarquons que ce milieu est très proche du Sabouraud au 1/10 avec sels minéraux, convenant à la sexualisation des Dermatophytes mis au point par Takashio (6).

Effectivement, ce dernier milieu permet la sexualisation 5 jours plus tard que le milieu proposé ci-dessus. Il a la même teneur en glucose, mais contient plus de $\mathrm{KH}_{2} \mathrm{PO}_{4}(1 \mathrm{~g} / 1)$ et de peptone $(1 \mathrm{~g} / 1)$.

On ensemence alors $L$. senegalensis sur un éventail de milieux dont la teneur en ces derniers produits s'étage de l'une à l'autre formule, on constate que, si on utilise la composition suivante: glucose : $2 \mathrm{~g} / 1$; néopeptone : $1 \mathrm{~g} / 1 ; \mathrm{KH}_{2} \mathrm{PO}_{4}$ : 1,5 g/1 ; $\mathrm{MgSO}_{4}, 7 \mathrm{H}_{2} \mathrm{O}: 0,5 \mathrm{~g} / 1$; gélose : $20 \mathrm{~g} / 1$, on obtient un optimum de périthèces mûrs dans les conditions physiques définies plus haut.

En conclusion, il est possible d'obtenir in vitro des périthèces du Leptosphaeria senegalensis sur un milieu relativement simple à condition de le cultiver entre 22 et 
$24^{\circ} \mathrm{C}$, sous un éclairement de lumière blanche fourni par des tubes fluorescents pendant 12 heures par jour: cet Ascomycète fait partie des espèces photo-induites.

\section{Bibliographie}

1. Baylet J., Camain R., Segrétain G. (1959): Identification des agents des Maduromycoses du Sénégal et de la Mauritanie. Description d'une espèce nouvelle. Bull. Soc. Pathol. Exot., 52, 448-477.

2. Dujardin L. : Etude des équilibres nutritifs favorables à la croissance et à la reproduction sexuée de deux Gymnoascaceae: Arachniotis albicans Apinis et Arthroderma vanbreuseghemi Takashio. Thèse Doctorat Sciences, $3^{e}$ cycle, Université des Sciences et Techniques de Lille, 1975.

3. Hall R. (1971) : Effects of carbon-nitrogen ratios on production of périthecia by Sordaria fimicola. Can. J. Microbiol., 17, 132-134.

4. Lazoste L., Dujardin L. (1972): La rep:oduction sexuée de Arachniotus albicans Apinis; influence de l'équilibre carbone-azote et du PH. Bull. Soc. Mycol., franc., 88, 5-14.

5. Lacoste L. : Biologie naturelle et culturale du genre Leptosphaeria Cesati et de Notaris. Déterminisme de la reproduction sexuelle. Thèse de Doctorat ès Sciences. Toulouse 1965.

6. Takashio M. (1973) : Etude des phénomènes de reproduction liés au vieillissement et au rajeunissement des cultures de champignons. Ann. Soc. belge Med. trop., 53, 427-580.

7. Westergaard M., Mitchell H. K. (1947) : Neurospora. A synthetic medium favoring sexual reproduction. Am. J. Bact., 34, 573-577. 